Hier können Sie CME-Punkte sammeln, und zwar a) für die Pflichtfortbildung aller Vertragsärzte und b) für freiwillige Fortbildungszertifikate, die viele Landesärztekammern anbieten.

Die folgenden Multiple-Choice-Fragen beziehen sich thematisch auf den vorangegangenen MMW-

Fortbildungsbeitrag auf den Seiten 28-31. Hinweis: Die Antworten auf die CME-Fragen ergeben sich nur

zum Teil direkt aus dem Text. Finige Fragen beruhen auf medizinischem Basiswissen!

Wenn Sie $70 \%$ der Fragen richtig beantworten, erhalten Sie 1 CME-Punkt.

Beantworten Sie $100 \%$ korrekt, erhalten Sie 2 CME-Punkte.

Einsendeschluss für die Teilnahme im Internet (www.cme-punkt.de): 15. Juni 2007

Einsendeschluss für die Teilnahme per Brief: 29. März 2007
Fortschritte der Medizin

CME-Herausgeber- und Review-Board: Dr. H. J. K. Barwitz, Prof. Dr. A. Berghaus, Prof. Dr. Dr. h.c. Th. Brandt, Prof. Dr. W. G. Daniel, Prof. Dr. W. Eisenmenger, Prof. Dr. K. Friese, Prof. Dr. H. S. Füessl, Prof. Dr. B. Göke, Prof. Dr. R. Gradinger, Prof. Dr. Dr. h.c. A. Hofstetter, Prof. Dr. H. Holzgreve, Prof. Dr. A. Imdahl, Prof. Dr. K. Krüger, Prof. Dr. H.-J. Möller, Prof. Dr. Dr. h.c. G. Plewig, Prof. Dr. D. Reinhardt, U. Weigeldt.

\title{
Fragen zum Thema „Pneumologische Diagnostik“
}

\section{- 1. Welche Aussage ist falsch?}

A Die Prävalenz von Asthma bronchiale und COPD beträgt zwischen 5 und $8 \%$.

B Weniger als 50\% der Erwachsenen mit Asthma bronchiale haben jemals eine Lungenfunktionsprüfung erhalten.

C Die Spirometrie sollte eine Standarddiagnostik in der Abklärung von Luftnot sein.

D Eine normale Lungenfunktion schließt eine pulmonale Ursache der Luftnot aus.

E Bei Rauchern treten KHK und COPD nicht selten gemeinsam auf.

\section{- 2. Was ist der größte Vorteil der Body-} plethysmografie?

A Es handelt sich um ein einfaches Untersuchungsverfahren, das in jeder Praxis durchgeführt werden kann.

B Die Vergütung ist am besten.

C Man ist nicht auf die Mitarbeit des Patienten angewiesen.

D Man benötigt kein Personal für die Untersuchung.

E Die Investitionskosten für das Gerät sind niedrig.

\section{- 3. Welche Aussage ist richtig?}

A Disease-Management-Programme für Asthma bronchiale und COPD stehen kurz vor der Einführung.

B Eine Spirometrie ist Voraussetzung für die Einschreibung in das DMP „Asthma bronchiale".

C Asthma bronchiale und COPD liegen nie gemeinsam bei einem Patienten vor.

D Ein Asthma bronchiale zeigt keine Reversibilität im Bronchospasmolyse-Test.

E Bei unklarer Diagnose eines Asthma bronchiale hilft ein Steroidversuch nicht weiter.
4. Welche Aussage zum Peak-Flow ist falsch?

A Das PEF-Meter gehört in die Notfalltasche des Hausarztes.

B Zirkadiane Schwankungen des PEF über 20\% sind typisch für ein Asthma bronchiale.

C Der PEF hilft bei der Beurteilung des Schweregrades eines Asthma bronchiale.

D Der PEF kann aus der Fluss-Volumen-Kurve abgelesen werden.

E Der PEF ist bei einem Asthma cardiale deutlich niedriger als bei einem Asthma bronchiale.

\section{- 5. Welche Aussage ist falsch?}

A Feines inspiratorisches Knistern kann auf eine Lungenfibrose hinweisen.

B Ein Pneumothorax ist eine Differenzialdiagnose bei akut einsetzender Luftnot.

C Die Auskultation kann einen Pneumothorax ausschließen.

D Ein Pneumothorax kann bei Patienten mit COPD oligosymptomatisch verlaufen.

E Ein einseitiger Pleuraerguss sollte diagnostisch weiter abgeklärt werden.

- 6. Welche Erkrankung ist keine Ursache eines chronisch persistierenden Hustens?

A Zwerchfellparese

B Chronische Bronchitis

C Gastroösophagealer Reflux

D Asthma bronchiale

E Chronische Sinusitis

\section{- 7. Welche Aussage zur Verordnung von} Inhalationssystemen trifft zu?

1. Der Verweis auf den Beipackzettel allein genügt nicht bei der Erstverordnung.

2. Die korrekte Inhalationstechnik muss im Verlauf überprüft werden.
3. Bei Dosieraerosolen ist die Koordination zwischen Sprühstoßauslösung und Beginn der Inhalation unproblematisch.

4. Bei Pulverinhalatoren sollte langsam und tief inhaliert werden.

5. Bei Pulverinhalatoren wird das eingefüllte Pulver durch den Inspirationsfluss des Patienten zerkleinert.

A Keine Aussage trifft zu.

B Nur Antwort 2 ist richtig.

C Antwort 1, 2 und 5 sind richtig.

D Alle Aussagen sind richtig.

E Antwort 3 und 4 sind richtig.

\section{- 8. Welcher Befund ist für ein Asthma} bronchiale charakteristisch?

A Es liegt immer eine Eosinophilie vor.

B Es sind nur junge Patienten betroffen.

C Es handelt sich fast immer um Nichtraucher.

D Die Patienten weisen zirkadiane Schwankungen bei der Peak-Flow-Messung von über $20 \%$ auf.

E Die Patienten haben nur tagsüber Husten.

- 9. Welche Erkrankung führt nicht zu einem Abfall der Sauerstoffsättigung?

A Akute Linksherzinsuffizienz

B Ambulant erworbene Pneumonie

C Lungenfibrose

D Akute Lungenembolie

E Hyperventilationssyndrom

\section{Welche Untersuchung gehört nicht zu} hausärztlichen pneumologischen Diagnostik?

A Auskultation des Thorax

B Spirometrie

C Pulsoxymetrie

D Bodyplethysmografie

E Peak-Flow-Meter

\section{ANTWORTFORMULAR}

Ich versichere, alle Fragen ohne fremde Hilfe beantwortet zu haben.

Mit dem Einreichen dieses Fragebogens erklärt sich der Einreichende damit einverstanden, dass die angegebenen Daten zum Zweck der Teilnahmebestätigung gespeichert und be erfolgreicher Teilnahme auch an den Einheitlichen Informationsverteiler (EIV) der Ärztekammern weitergegeben werden.
„Pneumologische Diagnostik“

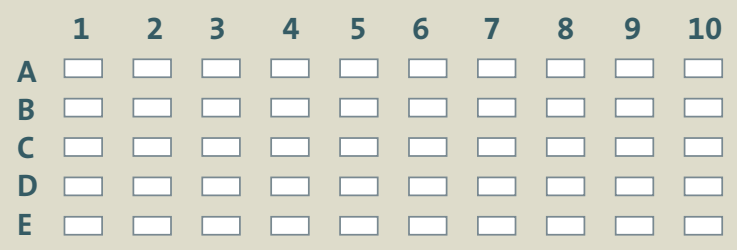

Bitte kreuzen Sie pro Frage je eine Antwort deutlich an.
Und so kommen Sie zu Ihren Punkten:

Teilnahme im Internet: unter www.cme-punkt.de. Dort führen wir für Sie ein elektronisches Punktekonto.

Teilnahme per Brief: Fragebogen ausfüllen und mit einem frankierten Rückumschlag an: Urban \& Vogel Medien und Medizin Verlagsgesellschaft, Redaktion MMW-Fortschritte de Medizin, Abt. Fortbildungszertifikat, Neumarkter Str. 43 81673 München. 